\title{
Aromatic Constituents from the Leaves of Actinidia arguta with Antioxidant and $\alpha$-Glucosidase Inhibitory Activity
}

\author{
Jong Hoon Ahn ${ }^{1, \dagger}$, Se Hwan Ryu ${ }^{1,+}$, Solip Lee ${ }^{1}$, Sang Won Yeon ${ }^{1}$, Ayman Turk ${ }^{1}$, Yoo Kyong Han ${ }^{2}$, \\ Ki Yong Lee ${ }^{2}$, Bang Yeon Hwang ${ }^{1}$ and Mi Kyeong Lee ${ }^{1, *(D)}$ \\ 1 College of Pharmacy, Chungbuk National University, Cheongju 28160, Korea; jhahn@dodamip.com (J.H.A.); \\ sehwan0188@chungbuk.ac.kr (S.H.R.); dudaos00@chungbuk.ac.kr (S.L.); \\ sangwon1352@chungbuk.ac.kr (S.W.Y.); aymanturk@chungbuk.ac.kr (A.T.); \\ byhwang@chungbuk.ac.kr (B.Y.H.) \\ 2 College of Pharmacy, Korea University, Sejong 47236, Korea; yookyong05@korea.ac.kr (Y.K.H.); \\ kylee11@korea.ac.kr (K.Y.L.) \\ * Correspondence: mklee@chungbuk.ac.kr; Tel.: +82-43-261-2818 \\ + These authors contributed equally to this work.
}

check for updates

Citation: Ahn, J.H.; Ryu, S.H.; Lee, S.; Yeon, S.W.; Turk, A.; Han, Y.K.; Lee,

K.Y.; Hwang, B.Y.; Lee, M.K.

Aromatic Constituents from the

Leaves of Actinidia arguta with Antioxidant and $\alpha$-Glucosidase Inhibitory Activity. Antioxidants 2021, 10, 1896. https://doi.org/10.3390/ antiox10121896

Academic Editor: Stanley Omaye

Received: 2 November 2021

Accepted: 25 November 2021

Published: 26 November 2021

Publisher's Note: MDPI stays neutral with regard to jurisdictional claims in published maps and institutional affiliations.

Copyright: (c) 2021 by the authors. Licensee MDPI, Basel, Switzerland. This article is an open access article distributed under the terms and conditions of the Creative Commons Attribution (CC BY) license (https:// creativecommons.org/licenses/by/ $4.0 /)$.
Abstract: As the leaf of Actinidia arguta has shown antioxidant activity, a study was conducted to identify the active ingredients. Forty-eight compounds were isolated from the leaves of $A$. arguta through various chromatographic techniques. Further characterization of the structures on the basis of 1D and 2D NMR and MS data identified several aromatic compounds, including phenylpropanoid derivatives, phenolics, coumarins, flavonoids and lignans. Among them, five compounds were newly reported, naturally occurring, and named argutosides A-D (1-4), which consist of phenylpropanoid glycosides that are conjugated with a phenolic moiety, and argutoside E (5), which is a coumarin glycoside that is conjugated with a phenylpropanoid unit. The isolated compounds showed good antioxidant and $\alpha$-glucosidase inhibitory activity with differences in activity depending on the structures. Molecular docking analysis demonstrated the interaction between the hydroxyl and carbonyl groups of compounds 1 and 5 with $\alpha$-glucosidase. Taken together, the leaves of A. arguta are rich in aromatic compounds with diverse structures. Therefore, the leaves of $A$. arguta and their aromatic components might be beneficial for oxidative stress and glucose-related diseases.

Keywords: Actinidia arguta; aromatic; argutosides A-E; antioxidant; $\alpha$-glucosidase; molecular docking analysis

\section{Introduction}

Oxidative stress is caused by the excessive production of reactive oxygen species (ROS) which bind to molecules in vivo and consequently alter their structures and functions. An antioxidant-related defense mechanism exists to protect against generated ROS. However, persistent oxidative stress by excessive production of ROS eventually leads to diverse severe diseases such as cancer, inflammation and metabolic diseases [1-3].

Diabetes is a metabolic disease with a high incidence worldwide. In diabetes, the blood glucose level increases due to the abnormal operation of insulin, which causes various complications and develops into a serious disease [4]. Various factors are known to be involved in the onset and progression of diabetes; oxidative stress is one such mediator $[5,6]$. The increased ROS attack the pancreas and interfere with the normal function of insulin $[7,8]$. In other words, oxidative stress and diabetes are mutually detrimental to each other $[9,10]$.

Accordingly, research into the development of a therapeutic agent for diabetes is being actively conducted. $\alpha$-Glucosidase is an intestinal enzyme which converts carbohydrates into single monosaccharides. Therefore, $\alpha$-glucosidase inhibitors are used for the treatment of diabetes and carbohydrate-mediated diseases [11,12]. Antioxidants are also used for the prevention and treatment of diabetes. 
Natural products are good sources for antioxidants and are widely used in the prevention and treatment of various diseases [13]. In particular, polyphenols are representative components with antioxidant action and are present in various plants. In addition, they also have therapeutic potential for metabolic diseases and have shown excellent results in various diabetes models [14,15].

Actinidia arguta, also called hardy kiwifruit or kiwiberry, has small fruits with a smooth green skin. Due to its cold-resistant characteristic, it can be cultivated in Northeast Asia [16,17]. The grains of $A$. arguta are mainly consumed as fresh fruits while the cooked leaves are used in the treatment of various diseases with antioxidant, antibacterial, antidiabetic and anti-inflammatory effects [18-20]. We recognized the importance of A. arguta as a native plant together with its biological activities and, thus, investigated the efficacy and ingredients of $A$. arguta. As a follow-up study on A. arguta leaves [21], the antioxidant and anti-diabetic effects of the extracts were confirmed. Further investigation into the bioactive constituents of $A$. arguta leaves resulted in the isolation of 48 compounds, including five new compounds. On the basis of 1D and 2D NMR and MS data, the structures of the isolated compounds were determined to be aromatic and included phenylpropanoid derivatives, phenolics, coumarins, flavonoids and lignans. The antioxidant and $\alpha$-glucosidase inhibitory activity of the isolated compounds were measured and their mechanism of action was analyzed using molecular docking analysis.

\section{Materials and Methods}

\subsection{Plant Material}

The leaves of A. arguta were obtained from a farm in Gwangyang, South Korea (GPS: DD 34.990714, 127.591508) in August 2016. After identification by the herbarium of the College of Pharmacy Chungbuk National University, voucher specimens (CBNU2016-AAL) were deposited in a specimen room of the herbarium.

\subsection{General Experimental Procedure}

The UV and IR spectra were obtained using Jasco UV-550 (JASCO, Tokyo, Japan) and Perkin-Elmer model LE599 (Perkin-Elmer, Waltham, MA, USA) spectrometer, respectively. A Bruker DRX 400 or $500 \mathrm{MHz}$ spectrometer (Bruker-Biospin, Karlsruhe, Germany) were used for the analysis of NMR signals using $\mathrm{CD}_{3} \mathrm{OD}$ as a solvent. ESIMS and HRESI-TOFMS data were obtained on LCQ Fleet and maXis 4G mass spectrometers (Bruker Daltonics, Bremen, Germany), respectively. Semi-preparative HPLC (Waters, Milford, MA, USA) was performed using a Waters 515 HPLC pump with a 996-photodiode array detector, and Waters Empower software using a Gemini-NX ODS-column $(150 \times 10.0 \mathrm{~mm}$ and $150 \times 21.2 \mathrm{~mm})$. Column chromatography procedures were performed using silica gel (200-400 mesh, Fisher Scientific, Waltham, MA, USA) and Sephadex LH-20 (25-100 $\mu$, Pharmacia Fine Chemical Industries Co., Uppsala, Sweden). Thin-layer chromatography (TLC) was performed using aluminum plates precoated with Kieselgel $60 \mathrm{~F}_{254}(0.25 \mathrm{~mm}$, Merck, Darmstadt, Germany).

\subsection{Extraction and Isolation}

The dried powder of $A$. arguta leaves $(4.0 \mathrm{~kg})$ was extracted with $80 \% \mathrm{MeOH}(30 \mathrm{~L} \times 2)$ at room temperature. The $\mathrm{MeOH}$ extract $(350.0 \mathrm{~g})$ was suspended in $\mathrm{H}_{2} \mathrm{O}(2 \mathrm{~L})$ and partitioned successively with $n$-hexane, $\mathrm{CH}_{2} \mathrm{Cl}_{2}$, EtOAc and $n$-BuOH (each $2 \mathrm{~L} \times 2$ ) for $24 \mathrm{~h}$.

The $\mathrm{CH}_{2} \mathrm{Cl}_{2}$ fraction (AALC, $24.1 \mathrm{~g}$ ) was chromatographed on silica gel and eluted with a mixture of $n$-hexane-EtOAc (100\% $n$-hexane to $100 \%$ EtOAc) to obtain fourteen subfractions (AALC1-C14). Subfraction C13 (3.2 g) was subjected to MPLC on RP-silica gel and eluted with mixtures of $\mathrm{MeOH}-\mathrm{H}_{2} \mathrm{O}(5 \% \mathrm{MeOH}$ to $100 \% \mathrm{MeOH})$ to give three subfractions (C13A-C13C). Compounds 12 and $\mathbf{4 3}$ were purified from C13B and C13C, respectively, by semi-preparative HPLC and eluted with acetonitrile- $\mathrm{H}_{2} \mathrm{O}$ (20:80). Compounds 41, 42 and 47 were purified from C14 F by Sephadex LH-20 and eluted with MeOH followed by semi-preparative HPLC and elution with acetonitrile- $\mathrm{H}_{2} \mathrm{O}$ (20:80). 
The EtOAc fraction (AALE, $24.4 \mathrm{~g}$ ) was chromatographed on silica gel and eluted with a mixture of $\mathrm{CH}_{2} \mathrm{Cl}_{2}-\mathrm{MeOH}$ by step gradient $\left(100 \% \mathrm{CH}_{2} \mathrm{Cl}_{2}\right.$ to $\left.100 \% \mathrm{MeOH}\right)$ to obtain eleven subfractions (AALE1-E11). Subfraction E4 (2.5 g) was subjected to MPLC on RP-silica gel and eluted with mixtures of $\mathrm{MeOH}-\mathrm{H}_{2} \mathrm{O}(5 \%$ to $100 \% \mathrm{MeOH})$ to give six subfractions (E4A-E4F). Subfraction E4C was separated into two subfractions (E4F1-E4F2) by Sephadex LH-20 (MeOH). Compounds 9, 10, 13, 14 and 20 were purified from E4C2 by semi-preparative HPLC (acetonitrile- $\mathrm{H}_{2} \mathrm{O}, 20: 80$ ). E5 (2.9 g) was subjected to MPLC on RP-silica gel (mixtures of $\mathrm{MeOH}-\mathrm{H}_{2} \mathrm{O}, 5 \%$ to $100 \% \mathrm{MeOH}$ ) to give eight subfractions (E5A-E5H). Sephadex LH-20 column chromatography $(\mathrm{MeOH})$ of E5B, E5C, E5G and E5H gave compounds 11, 8, 31 and 22, respectively. Compounds 17 and 18 were isolated from E5D by Sephadex LH-20 (MeOH) followed by semi-preparative HPLC and elution with acetonitrile- $\mathrm{H}_{2} \mathrm{O}$ (30:70). Subfraction E5F was separated by Sephadex LH-20 (MeOH) to obtain E5F1 and E5F2, which gives compounds 32 and 29, respectively, by semi-preparative HPLC and elution with acetonitrile- $\mathrm{H}_{2} \mathrm{O}(30: 70)$.

Subfraction E6 (3.2 g) was subjected to MPLC on RP-silica gel and eluted with mixtures of $\mathrm{MeOH}-\mathrm{H}_{2} \mathrm{O}(5 \% \mathrm{MeOH}$ to $100 \% \mathrm{MeOH})$ to give eight subfractions (E6A-E6H). Compounds 12 and 15 were purified from E6B by semi-preparative HPLC (acetonitrile$\left.\mathrm{H}_{2} \mathrm{O}, 50: 50\right)$. Compounds 16 and 19 were purified from E6C by Sephadex LH-20 (MeOH) followed by semi-preparative HPLC (acetonitrile- $\mathrm{H}_{2} \mathrm{O}, 50: 50$ ). Compounds 30 and 25 were purified from E6D and E6F, respectively, by Sephadex LH-20 (MeOH). E7 (2.6 g) was subjected to MPLC on RP-silica gel and eluted with mixtures of $\mathrm{MeOH}-\mathrm{H}_{2} \mathrm{O}(10 \% \mathrm{MeOH}$ to $100 \% \mathrm{MeOH}$ ) to give nine subfractions (E7A-E7I). E7D was separated to obtain E7D1 by Sephadex LH-20 and eluted with MeOH. Compound 40 was purified from E7D1 by semi-preparative HPLC and eluted with acetonitrile- $\mathrm{H}_{2} \mathrm{O}$ (30:70).

Subfraction E8 (4.2 g) was subjected to MPLC on RP-silica gel and eluted with mixtures of $\mathrm{MeOH}-\mathrm{H}_{2} \mathrm{O}(5 \%$ to $100 \% \mathrm{MeOH})$ to give nine subfractions (E8A-E8I). E8C, E8D and E8E were subjected to Sephadex LH-20 (MeOH) to give compound 37, 36 and 38, respectively. Subfraction E8G was separated by Sephadex LH-20 (MeOH) to obtain E8G1 and E8G2, which gives compounds 44 and 39, respectively, by semi-preparative HPLC and elution with MeCN-H2O (20:80). E8I (2.9 g) was subjected to Sephadex LH-20 (MeOH) to give four subfractions (E8I1-E8I4). Compounds 45 and 46 were isolated from E8I2 by semipreparative HPLC (acetonitrile- $\mathrm{H}_{2} \mathrm{O}, 18: 82$ ). Semi-preparative HPLC (acetonitrile- $\mathrm{H}_{2} \mathrm{O}$, 18:82) of E8I4 gives compounds 1, 5, 6, 7 and 28.

Subfraction E9 (5.0 g) was subjected to MPLC on RP-silica gel and eluted with mixtures of $\mathrm{MeOH}-\mathrm{H}_{2} \mathrm{O}(5 \%$ to $100 \% \mathrm{MeOH})$ to give ten subfractions (E9A-E9J). Compounds 33, 23, 34 and 35 were isolated from E9E, E9H, E9I and E9J, respectively, by Sephadex LH-20 $(\mathrm{MeOH})$. Subfraction E9H was subjected to Sephadex LH-20 (MeOH) to give 3 subfractions (E9H1-E9H3). Compounds 2 and 3 were purified from E9H2 by semi-preparative HPLC (acetonitrile- $\left.\mathrm{H}_{2} \mathrm{O}, 20: 80\right)$. Subfraction E9I was subjected to Sephadex LH-20 (MeOH) to give 4 subfractions (E9I1-E9I4). Compounds 4 and 48 were obtained from E9I2 by semipreparative HPLC (acetonitrile- $\mathrm{H}_{2} \mathrm{O}, 23: 77$ ). Compounds 24, 26 and 27 were purified from E9I3 by semi-preparative HPLC $\left(\mathrm{MeCN}-\mathrm{H}_{2} \mathrm{O}, 20: 80\right)$.

\subsubsection{Argutoside A (1)}

Brown syrup; IR $v_{\max } 3411,1664 \mathrm{~cm}^{-1} ;{ }^{1} \mathrm{H}-\mathrm{NMR}\left(400 \mathrm{MHz}, \mathrm{CD}_{3} \mathrm{OD}\right)$ and ${ }^{13} \mathrm{C}-\mathrm{NMR}$ (100 MHz, CD 3 OD), see Tables 1 and 2, Figures S1-S4; HRESI-TOF-MS (positive mode) $\mathrm{m} / \mathrm{z} 511.1210$ (calcd. for $\mathrm{C}_{24} \mathrm{H}_{24} \mathrm{NaO}_{11}, 511.1216$, Figure S5).

\subsubsection{Argutoside B (2)}

Brown syrup; IR $v_{\max } 3411,1631 \mathrm{~cm}^{-1} ;{ }^{1} \mathrm{H}-\mathrm{NMR}\left(400 \mathrm{MHz}, \mathrm{CD}_{3} \mathrm{OD}\right)$ and ${ }^{13} \mathrm{C}-\mathrm{NMR}$ (100 MHz, CD 3 OD), see Tables 1 and 2, Figures S6-S9; HRESI-TOF-MS (positive mode) $\mathrm{m} / \mathrm{z}$ 485.1418 (calcd. for $\mathrm{C}_{23} \mathrm{H}_{26} \mathrm{NaO}_{10}, 485.1424$, Figure $\mathrm{S} 10$ ). 


\subsubsection{Argutoside C (3)}

Brown syrup; IR $v_{\max } 3411,1666 \mathrm{~cm}^{-1} ;{ }^{1} \mathrm{H}-\mathrm{NMR}\left(400 \mathrm{MHz}, \mathrm{CD}_{3} \mathrm{OD}\right)$ and ${ }^{13} \mathrm{C}-\mathrm{NMR}$ $\left(100 \mathrm{MHz}, \mathrm{CD}_{3} \mathrm{OD}\right)$, see Tables 1 and 2, Figures S11-S14: ESIMS m/z 485 [M + Na] $]^{+}$; HRESI-TOFMS (positive mode) $m / z 485.1418$ ([M + Na] $]^{+}$calcd. for $\mathrm{C}_{23} \mathrm{H}_{26} \mathrm{NaO}_{10}, 485.1424$, Figure S15).

\subsubsection{Argutoside D (4)}

Brown syrup; IR $v_{\max } 3423,1666 \mathrm{~cm}^{-1} ;{ }^{1} \mathrm{H}-\mathrm{NMR}\left(400 \mathrm{MHz}, \mathrm{CD}_{3} \mathrm{OD}\right)$ and ${ }^{13} \mathrm{C}-\mathrm{NMR}$ (100 MHz, CD $\mathrm{CD}_{3} \mathrm{OD}$ ), see Tables 1 and 2, Figures S16-S19; HRESI-TOF-MS m/z 529.1680 (calcd. for $\mathrm{C}_{25} \mathrm{H}_{30} \mathrm{NaO}_{11}, 529.1686$, Figure S20).

\subsubsection{Argutoside E (5)}

Brown syrup; IR $v_{\max } 3419,1660 \mathrm{~cm}-1 ;{ }^{1} \mathrm{H}-\mathrm{NMR}\left(400 \mathrm{MHz}\right.$, DMSO- $\left.d_{6}\right)$ and ${ }^{13} \mathrm{C}-\mathrm{NMR}$ $\left(100 \mathrm{MHz}\right.$, DMSO- $d_{6}$ ), see Table 3 and Figures S21-S24; HRESI-TOF-MS m/z 525.1003 (calcd. for $\mathrm{C}_{24} \mathrm{H}_{22} \mathrm{NaO}_{12}, 525.1009$, Figure S25).

\subsection{Measurement of $\alpha$-Glucosidase Activity}

The inhibitory effect on $\alpha$-glucosidase was measured using $\alpha$-glucosidase (from Saccharomyces cerevisiae (EC 3.2.1.20) [21]. A test sample was mixed with $80 \mu \mathrm{L}$ enzyme buffer and $10 \mu \mathrm{L} \alpha$-glucosidase and incubated for $15 \mathrm{~min}$ at $37^{\circ} \mathrm{C}$. Then, after the addition of $10 \mu \mathrm{L} p$-nitrophenyl $\alpha$-D-glucopyranoside solution for enzyme reaction, the amount of $p$-nitrophenol that was cleaved by the enzyme was determined by measuring the absorbance at $405 \mathrm{~nm}$ in a 96-well microplate reader. Acarbose was used as a positive control.

\subsection{Measurement of DPPH Radical Scavenging Activity}

The antioxidant activity was evaluated by measuring the free radical scavenging activity using DPPH as previously reported [20]. In brief, freshly prepared DPPH solution was mixed with the samples. The mixture was reacted at room temperature for $10 \mathrm{~min}$, and the absorbance was measured at $550 \mathrm{~nm}$. Ascorbic acid was used as a positive control.

\subsection{Molecular Docking Studies}

SYBYL-X 2.1.1 (Tripos Ltd., St. Louis, MO, USA) with crystal structures of N-terminal subunit (NtMGAM; PDB-ID: 2QMJ) and C-terminal subunit (CtMGAM; PDB-ID: 3TOP) of human maltase-glucoamylase (MGAM) were used, respectively, for molecular docking studies of active compounds [19].

\section{Results}

\subsection{Structural Elucidation}

Chromatographic separation of the EtOAc fraction of A. arguta resulted in the isolation of five new compounds (1-5) together with forty-three known compounds (6-48) (Figure 1). 

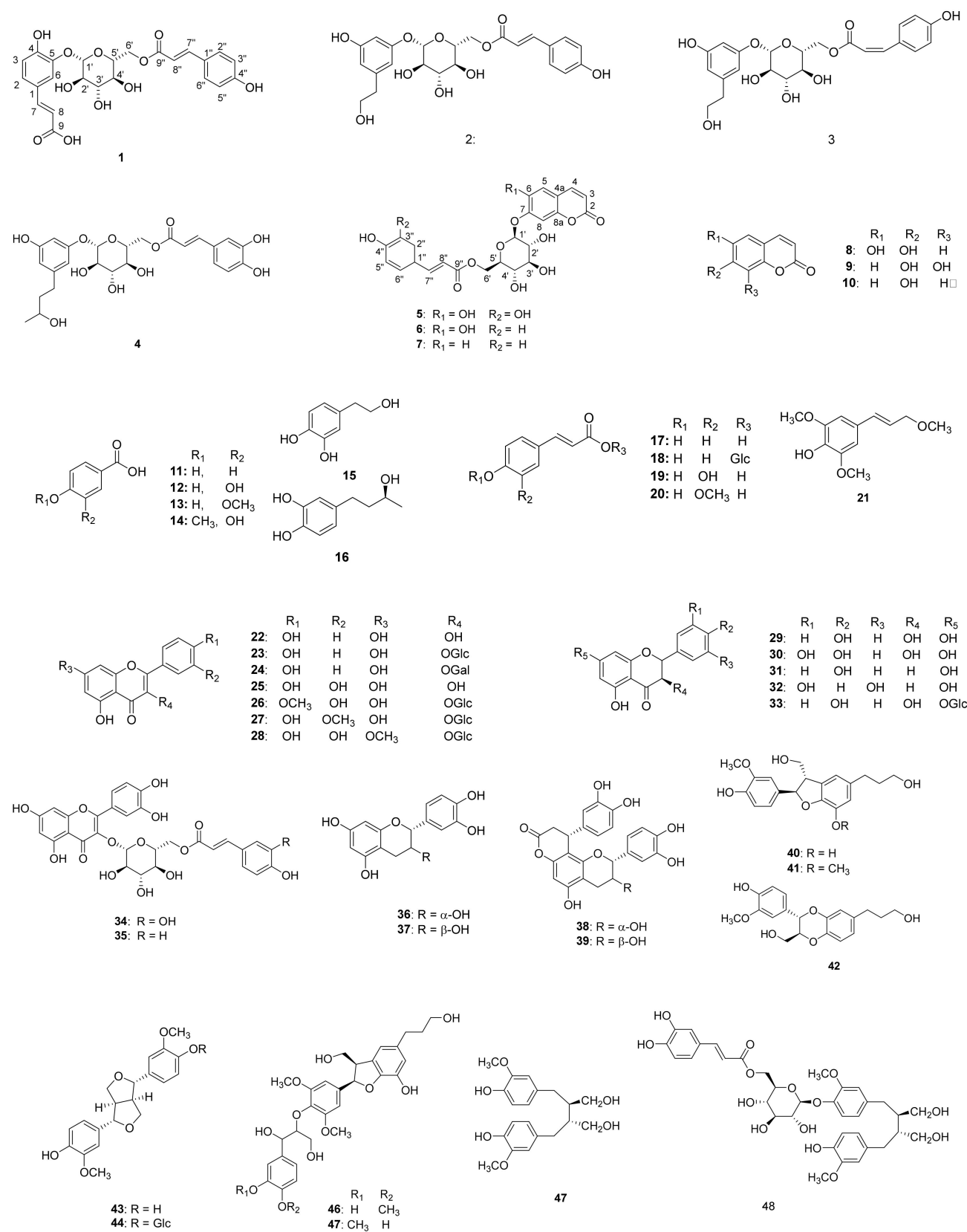

Figure 1. Chemical structures of compounds 1-48 from the leaves of A. arguta.

\subsubsection{Structural Determination of New Compounds}

Compound $\mathbf{1}$ (Tables 1 and 2) was isolated as a brown syrup and the molecular formula was deduced as $\mathrm{C}_{24} \mathrm{H}_{24} \mathrm{O}_{11}$ from the HRESI-TOF-MS $\left(\mathrm{m} / z 511.1210[\mathrm{M}+\mathrm{Na}]^{+}\right.$, calcd. for $\left.\mathrm{C}_{24} \mathrm{H}_{24} \mathrm{NaO}_{11}, 511.1216\right)$ and ${ }^{13} \mathrm{C}-\mathrm{NMR}$ data. The IR spectrum showed typical absorption bands of hydroxy and carbonyl groups at 3411 and $1664 \mathrm{~cm}^{-1}$, respectively. The ${ }^{1} \mathrm{H}-\mathrm{NMR}$ spectrum of compound 1 showed typical signals for a glucosyl anomeric proton in the $\beta$-configuration at $\delta_{\mathrm{H}} 4.92\left(1 \mathrm{H}, \mathrm{d}, J=7.6 \mathrm{~Hz}, \mathrm{H}-1^{\prime}\right)$. The presence of a glucosyl moiety was also confirmed by the glucosyl carbon signals at $101.9\left(\mathrm{C}-1^{\prime}\right), 73.3\left(\mathrm{C}-2^{\prime}\right), 76.0\left(\mathrm{C}-3^{\prime}\right), 70.6$ $\left.\left(\mathrm{C}-4^{\prime}\right), 74.3\left(\mathrm{C}-5^{\prime}\right), 63.5\left(\mathrm{C}-6^{\prime}\right)\right]$. In the aromatic regions of the ${ }^{1} \mathrm{H}$ - and ${ }^{13} \mathrm{C}-\mathrm{NMR}$, signals for a 1,3,4-trisubstituted aromatic ring at $\left[\delta_{\mathrm{H}} 7.41(1 \mathrm{H}, \mathrm{d}, J=2.0 \mathrm{~Hz}, \mathrm{H}-2), 7.17(1 \mathrm{H}, \mathrm{d}\right.$, $J=8.4,2.0 \mathrm{~Hz}, \mathrm{H}-6), 6.88(1 \mathrm{H}, \mathrm{d}, J=8.4 \mathrm{~Hz}, \mathrm{H}-5) ; \delta_{\mathrm{C}} 126.6$ (C-1), $116.0(\mathrm{C}-2), 145.4(\mathrm{C}-3)$, $149.4(\mathrm{C}-4), 116.1(\mathrm{C}-5), 124.4(\mathrm{C}-6)]$ and a 1,4-disubstituted aromatic ring at $\left[\delta_{\mathrm{H}} 7.42(2 \mathrm{H}, \mathrm{d}\right.$, $\left.J=8.8 \mathrm{~Hz}, \mathrm{H}-3^{\prime \prime}, 5^{\prime \prime}\right), 6.80\left(2 \mathrm{H}, \mathrm{d}, J=8.8 \mathrm{~Hz}, \mathrm{H}-2^{\prime \prime}, 6^{\prime \prime}\right) ; \delta_{\mathrm{C}} 125.7\left(\mathrm{C}-1^{\prime \prime}\right), 115.4\left(\mathrm{C}-2^{\prime \prime}, 6^{\prime \prime}\right), 129.9$ $\left.\left(\mathrm{C}-3^{\prime \prime}, 5^{\prime \prime}\right), 159.9\left(\mathrm{C}-4^{\prime \prime}\right)\right]$ were observed. The ${ }^{1} \mathrm{H}-\mathrm{NMR}$ spectrum also revealed two pairs of 
olefinic groups in the trans configuration at $\left[\delta_{\mathrm{H}} 7.58(1 \mathrm{H}, \mathrm{d}, J=15.6 \mathrm{~Hz}, \mathrm{H}-7), 6.33(1 \mathrm{H}, \mathrm{d}\right.$, $J=15.6 \mathrm{~Hz}, \mathrm{H}-8) ; \delta \mathrm{c} 144.7(\mathrm{C}-7), 116.1(\mathrm{C}-8)]$ and $\left[\delta_{\mathrm{H}} 7.61\left(1 \mathrm{H}, \mathrm{d}, J=16.0 \mathrm{~Hz}, \mathrm{H}-7^{\prime \prime}\right), 6.34\right.$ $\left.\left(1 \mathrm{H}, \mathrm{d}, J=16.0 \mathrm{~Hz}, \mathrm{H}-8^{\prime \prime}\right) ; \delta \mathrm{c} 145.7\left(\mathrm{C}-7^{\prime \prime}\right), 113.2\left(\mathrm{C}-8^{\prime \prime}\right)\right]$, respectively. Additionally, signals for two carbonyl carbons $\left[\delta_{\mathrm{C}} 169.6\right.$ and 167.8] were observed in the ${ }^{13} \mathrm{C}-\mathrm{NMR}$ spectrum. These signals were assigned to the trans-caffeoyl group and the trans-coumaroyl group based on the HMBC correlations between H-7/C-1, H-7/C-9 and H-2" /C-7", H-7" /C-9", respectively (Figure 2). Therefore, compound 1 was suggested to consist of a glucose, a trans-caffeoyl group and a trans-coumaroyl group. The connections between these moieties were determined by HMBC correlation. The HMBC correlations from $\mathrm{H}-1^{\prime}$ of a glucose to $\mathrm{C}-5$ of a trans-caffeoyl group, and from $\mathrm{H}-6^{\prime}$ of a glucose to C-9" of a trans-coumaroyl group suggested the linkages of a trans-caffeoyl group to a glucose and a glucose to a trans-coumaroyl group. Combined with the above-mentioned data, compound $\mathbf{1}$ was elucidated as shown and named argutoside A.

Table 1. ${ }^{1} \mathrm{H}-\mathrm{NMR}$ spectroscopic data for compounds $1-4\left(\mathrm{CD}_{3} \mathrm{OD}\right)$.

\begin{tabular}{ccccc}
\hline Title 1 & $\mathbf{1}$ & $\mathbf{2}$ & $\mathbf{3}$ & $\mathbf{4}$ \\
\hline 2 & $7.41(\mathrm{~d}, 2.0)$ & $6.78(\mathrm{~s})$ & $6.78(\mathrm{~s})$ & $6.76(\mathrm{~s})$ \\
4 & - & $6.78(\mathrm{~s})$ & $6.78(\mathrm{~s})$ & $6.76(\mathrm{~s})$ \\
5 & $6.88(\mathrm{~d}, 8.4)$ & - & - & - \\
6 & $7.17(\mathrm{dd}, 8.4,2.0)$ & $7.04(\mathrm{~s})$ & $7.04(\mathrm{~s})$ & $7.00(\mathrm{~s})$ \\
7 & $7.58(\mathrm{~d}, 15.6)$ & $2.67(2 \mathrm{H}, \mathrm{t}, 7.2)$ & $2.71(2 \mathrm{H}, \mathrm{t}, 7.2)$ & $2.53(2 \mathrm{H}, \mathrm{m})$ \\
8 & $6.33(\mathrm{~d}, 15.6)$ & $3.67(2 \mathrm{H}, \mathrm{t}, 7.2)$ & $3.70(2 \mathrm{H}, \mathrm{t}, 7.2)$ & $1.63(2 \mathrm{H}, \mathrm{m})$ \\
9 & - & - & - & $3.62(\mathrm{~m})$ \\
10 & - & - & - & $1.11(3 \mathrm{H}, \mathrm{d}, 6.0)$ \\
$1^{\prime}$ & $4.92(\mathrm{~d}, 7.6)$ & $4.78(\mathrm{~d}, 7.6)$ & $4.76(\mathrm{~d}, 7.6)$ & $4.77(\mathrm{~d} .7 .2)$ \\
$2^{\prime}$ & $3.41-3.57(\mathrm{~m})$ & $3.40-3.54(\mathrm{~m})$ & $3.40-3.52(\mathrm{~m})$ & $3.41-3.54(\mathrm{~m})$ \\
$3^{\prime}$ & $3.41-3.57(\mathrm{~m})$ & $3.40-3.54(\mathrm{~m})$ & $3.40-3.52(\mathrm{~m})$ & $3.41-3.54(\mathrm{~m})$ \\
$4^{\prime}$ & $3.41-3.57(\mathrm{~m})$ & $3.40-3.54(\mathrm{~m})$ & $3.40-3.52(\mathrm{~m})$ & $3.41-3.54(\mathrm{~m})$ \\
$5^{\prime}$ & $3.82(\mathrm{~m})$ & $3.73(\mathrm{~m})$ & $3.67(\mathrm{~m})$ & $3.74(\mathrm{~m})$ \\
$6^{\prime}$ & $4.59(\mathrm{dd}, 12.0,2.0)$ & $4.60(\mathrm{dd}, 12.0,2.0)$ & $4.55(\mathrm{dd}, 12.0,2.0)$ & $4.59(\mathrm{dd}, 12.0,2.4)$ \\
& $4.38(\mathrm{dd}, 12.0,6.6)$ & $4.37(\mathrm{dd}, 12.0,6.6)$ & $4.33(\mathrm{dd}, 12.0,6.6)$ & $4.37(\mathrm{dd}, 12.0,6.8)$ \\
$2^{\prime \prime}$ & $6.80(\mathrm{~d}, 8.8)$ & $6.83(\mathrm{~d}, 8.8)$ & $6.73(\mathrm{~d}, 8.8)$ & $7.08(\mathrm{~d}, 1.6)$ \\
$3^{\prime \prime}$ & $7.42(\mathrm{~d}, 8.8)$ & $7.50(\mathrm{~d}, 8.8)$ & $7.66(\mathrm{~d}, 8.8)$ & - \\
$5^{\prime \prime}$ & $7.42(\mathrm{~d}, 8.8)$ & $7.50(\mathrm{~d}, 8.8)$ & $7.66(\mathrm{~d}, 8.8)$ & $6.80(\mathrm{~d}, 8.4)$ \\
$6^{\prime \prime}$ & $6.80(\mathrm{~d}, 8.8)$ & $6.83(\mathrm{~d}, 8.8)$ & $6.73(\mathrm{~d}, 8.8)$ & $6.97(\mathrm{dd}, 8.4,1.6)$ \\
$7^{\prime \prime}$ & $7.61(\mathrm{~d}, 16.0)$ & $7.68(\mathrm{~d}, 16.0)$ & $6.93(\mathrm{~d}, 12.8)$ & $7.60(\mathrm{~d}, 15.6)$ \\
$8^{\prime \prime}$ & $6.34(\mathrm{~d}, 16.0)$ & $6.41(\mathrm{~d}, 16.0)$ & $5.83(\mathrm{~d}, 12.8)$ & $6.33(\mathrm{~d}, 15.6)$ \\
\hline
\end{tabular}

Compound 2 (Tables 1 and 2) was purified as a brown syrup. The molecular formula was deduced as $\mathrm{C}_{23} \mathrm{H}_{26} \mathrm{NaO}_{10}$ from the HRESI-TOF-MS $(\mathrm{m} / z 485.1418 \text { [M }+\mathrm{Na}]^{+}$, calcd. for $\left.\mathrm{C}_{23} \mathrm{H}_{26} \mathrm{NaO}_{10}, 485.1424\right)$ ), which was verified by its ${ }^{13} \mathrm{C}-\mathrm{NMR}$ data. Similar to compound $\mathbf{1}$, the presence of a glucose was easily deduced from glucosyl anomeric signals $\left[\delta_{\mathrm{H}} 4.78\right.$ $\left.\left(1 \mathrm{H}, \mathrm{d}, J=7.6 \mathrm{~Hz}, \mathrm{H}-1^{\prime}\right) ; \delta_{\mathrm{C}} 102.9\right]$. The presence of a trans-coumaroyl group was also suggested by the signals at $\left[\delta_{\mathrm{H}} 6.83\left(2 \mathrm{H}, \mathrm{d}, J=8.8 \mathrm{~Hz}, \mathrm{H}-2^{\prime \prime}, 6^{\prime \prime}\right), 7.50(2 \mathrm{H}, \mathrm{d}, J=8.8 \mathrm{~Hz}\right.$, H-3", 5"), $7.68\left(1 \mathrm{H}, \mathrm{d}, J=16.0 \mathrm{~Hz}, \mathrm{H}-7^{\prime \prime}\right), 6.41\left(1 \mathrm{H}, \mathrm{d}, J=16.0 \mathrm{~Hz}, \mathrm{H}-8^{\prime \prime}\right) ; \delta_{\mathrm{C}} 125.7$ (C-1"), 115.4 (C-2", 6"), 129.9 (C-3", 5"), 160.0 (C-4"), 145.6 (C-7"), 113.4 (C-8"), 167.6 (C-9")] together with the $\mathrm{HMBC}$ correlations. Besides the aforementioned signals for a glucose and trans-coumaroyl group, signals for a 1,3,5-trisubstituted aromatic ring $\left[\delta_{\mathrm{H}} 6.78(2 \mathrm{H}\right.$, s, H-2, 4), $7.04(1 \mathrm{H}, \mathrm{s}, \mathrm{H}-6) ; \delta_{\mathrm{C}} 130.7$ (C-1), 123.9 (C-2), 145.1 (C-3), 115.5 (C-4), 145.3 (C-5), 118.1 (C-6)] two methylene $\left[\delta_{\mathrm{H}} 2.67(2 \mathrm{H}, \mathrm{t}, J=7.2 \mathrm{~Hz}, \mathrm{H}-7), 3.67(2 \mathrm{H}, \mathrm{t}, J=7.2 \mathrm{~Hz}, \mathrm{H}-8)\right.$; $\left.\delta_{\mathrm{C}} 38.2(\mathrm{C}-7), 62.9(\mathrm{C}-8)\right]$, and an oxygenated methine $\left[\delta_{\mathrm{H}} 3.62(1 \mathrm{H}, \mathrm{m}, \mathrm{H}-9) ; \delta_{\mathrm{C}} 66.3\right]$ were observed in ${ }^{1} \mathrm{H}$ - and ${ }^{13} \mathrm{C}-\mathrm{NMR}$ together with HSQC spectrum. These additional signals were assigned to a 3,5-dihydroxyphenylethanol group based on the correlations between $\mathrm{H}$ 2/C-7 and H-8/C-7 in the HMBC spectrum. The positions of a 3, 5-dihydroxyphenylethanol group and a trans-coumaroyl group were determined to be $\mathrm{C}-1^{\prime}$ and $\mathrm{C}-6^{\prime}$, respectively, from the HMBC correlations of $\mathrm{H}-1^{\prime} / \mathrm{C}-3$ and $\mathrm{H}-6^{\prime} / \mathrm{C}-9^{\prime \prime}$. Consequently, the structure 
of compound 2 was defined as shown and named argutoside B. The ${ }^{1} \mathrm{H}-$ and ${ }^{13} \mathrm{C}-\mathrm{NMR}$ spectra of 3 (Tables 1 and 2) were similar to those of compound 2, with the difference being the replacement of the trans-olefinic protons with a large coupling constant $\left[\delta_{\mathrm{H}} 7.68\right.$ $\left.\left(1 \mathrm{H}, \mathrm{d}, J=16.0 \mathrm{~Hz}, \mathrm{H}-7^{\prime \prime}\right), 6.41\left(1 \mathrm{H}, \mathrm{d}, J=16.0 \mathrm{~Hz}, \mathrm{H}-8^{\prime \prime}\right)\right]$ by cis-olefinic protons with a smaller coupling constant $\left[\delta_{\mathrm{H}} 6.93\left(1 \mathrm{H}, \mathrm{d}, J=12.8 \mathrm{~Hz}, \mathrm{H}-7^{\prime}\right), 5.86\left(1 \mathrm{H}, \mathrm{d}, J=12.8 \mathrm{~Hz}, \mathrm{H}-8^{\prime}\right)\right]$. Therefore, the structure of compound 3 was defined as shown and named argutoside $C$.

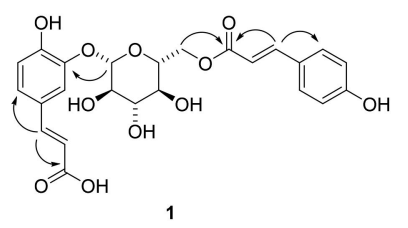

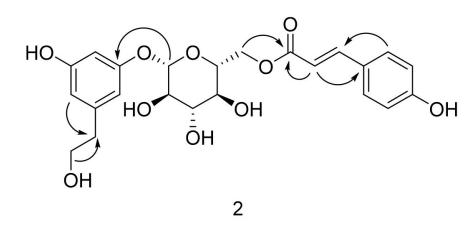

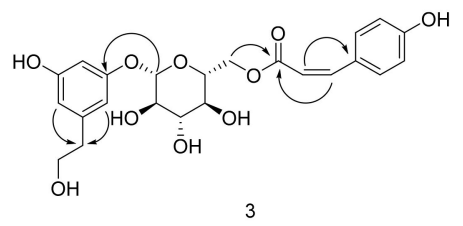

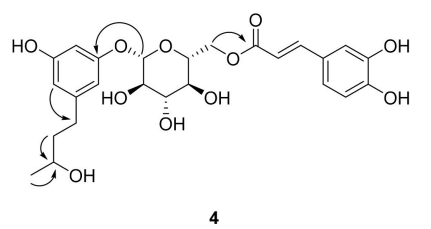

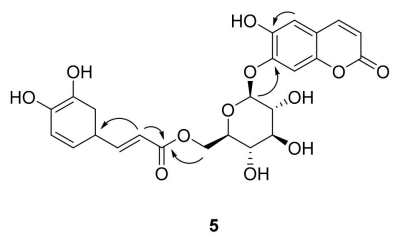

Figure 2. Key HMBC correlations $(\rightarrow)$ of new compounds 1-5.

Table 2. ${ }^{13} \mathrm{C}-\mathrm{NMR}$ spectroscopic data for compounds $\mathbf{1 - 4}\left(\mathrm{CD}_{3} \mathrm{OD}\right)$.

\begin{tabular}{ccccc}
\hline Carbon NO. & $\mathbf{1}$ & $\mathbf{2}$ & $\mathbf{3}$ & $\mathbf{4}$ \\
\hline 1 & 126.6 & 130.7 & 130.6 & 133.9 \\
2 & 116.0 & 123.9 & 123.9 & 123.2 \\
3 & 145.4 & 145.1 & 145.1 & 144.8 \\
4 & 149.4 & 115.5 & 115.5 & 115.5 \\
5 & 116.1 & 145.3 & 145.3 & 145.1 \\
6 & 124.4 & 118.1 & 118.1 & 117.5 \\
7 & 144.7 & 38.2 & 38.2 & 31.1 \\
8 & 115.5 & 62.9 & 63.0 & 40.6 \\
9 & 169.6 & - & - & 66.3 \\
10 & - & - & - & 22.1 \\
$1^{\prime}$ & 101.9 & 102.9 & 102.9 & 102.9 \\
$2^{\prime}$ & 73.3 & 73.4 & 73.4 & 73.4 \\
$3^{\prime}$ & 76.0 & 76.0 & 76.0 & 76.0 \\
$4^{\prime}$ & 70.6 & 70.4 & 70.3 & 70.4 \\
$5^{\prime}$ & 74.3 & 74.4 & 74.3 & 74.4 \\
$6^{\prime}$ & 63.5 & 63.3 & 63.0 & 63.3 \\
$1^{\prime \prime}$ & 125.7 & 125.7 & 126.1 & 126.2 \\
$2^{\prime \prime}$ & 115.4 & 115.4 & 114.4 & 113.7 \\
$3^{\prime \prime}$ & 129.9 & 129.9 & 132.4 & 145.5 \\
$4^{\prime \prime}$ & 159.9 & 160.0 & 158.7 & 148.3 \\
$5^{\prime \prime}$ & 129.9 & 129.9 & 132.4 & 115.1 \\
$6^{\prime \prime}$ & 115.4 & 115.4 & 114.4 & 121.8 \\
$7^{\prime \prime}$ & 145.7 & 145.6 & 144.3 & 146.0 \\
$8^{\prime \prime}$ & 113.2 & 113.4 & 114.7 & 113.3 \\
$9^{\prime \prime}$ & 167.8 & 167.6 & 166.6 & 167.6 \\
\hline
\end{tabular}


Compound 4 (Tables 1 and 2) was purified as brown syrup. The molecular formula was deduced as $\mathrm{C}_{25} \mathrm{H}_{30} \mathrm{NaO}_{11}$ from the HRESI-TOF-MS $\left(\mathrm{m} / z 529.1680[\mathrm{M}+\mathrm{Na}]^{+}\right.$, calcd. for $\mathrm{C}_{25} \mathrm{H}_{30} \mathrm{NaO}_{11}, 529.1686$ ), which was verified by its ${ }^{13} \mathrm{C}-\mathrm{NMR}$ data. Similar to compounds $1-3$, the presence of a glucose was easily deduced from glucosyl anomeric signals $\left[\delta_{\mathrm{H}}\right.$ $\left.4.77\left(1 \mathrm{H}, \mathrm{d}, J=7.2 \mathrm{~Hz}, \mathrm{H}-1^{\prime}\right) ; \delta_{\mathrm{C}} 102.9\right]$. The presence of a trans-caffeoyl group was also suggested from the signals at $\left[\delta_{\mathrm{H}} 7.08\left(1 \mathrm{H}, \mathrm{d}, J=1.6 \mathrm{~Hz}, \mathrm{H}-2^{\prime \prime}\right), 6.80(1 \mathrm{H}, \mathrm{d}, J=8.4,1.6\right.$ $\left.\mathrm{Hz}, \mathrm{H}-5^{\prime \prime}\right), 6.97\left(1 \mathrm{H}, \mathrm{d}, J=8.4 \mathrm{~Hz}, \mathrm{H}-6^{\prime \prime}\right), 7.60\left(1 \mathrm{H}, \mathrm{d}, J=15.6 \mathrm{~Hz}, \mathrm{H}-7^{\prime \prime}\right), 6.33(1 \mathrm{H}, \mathrm{d}, J$ $\left.=15.6 \mathrm{~Hz}, \mathrm{H}-8^{\prime \prime}\right) ; \delta_{\mathrm{C}} 126.2\left(\mathrm{C}-1^{\prime \prime}\right), 113.7\left(\mathrm{C}-2^{\prime \prime}\right), 145.5$ (C-3"), $148.3\left(\mathrm{C}-4^{\prime \prime}\right), 115.1\left(\mathrm{C}-5^{\prime \prime}\right)$, 121.8 (C-6"), 146.0 (C-7"), $113.3\left(\mathrm{C}-8^{\prime \prime}\right), 167.6$ (C-9")] together with the HMBC correlations. Besides the aforementioned signals, signals for a 1,3,5-trisubstituted aromatic ring $\left[\delta_{\mathrm{H}} 6.76\right.$ (2H, s, H-2, 4), 7.00 (1H, s, H-6); $\delta_{\mathrm{C}} 133.9$ (C-1), 123.2 (C-2), 144.8 (C-3), 115.5 (C-4), 145.1 (C-5), $117.5(\mathrm{C}-6)]$, two methylene $\left[\delta_{\mathrm{H}} 2.53(2 \mathrm{H}, \mathrm{m}, \mathrm{H}-7), 1.63(2 \mathrm{H}, \mathrm{m}, \mathrm{H}-8) ; \delta_{\mathrm{C}} 31.1\right.$ (C-7), $40.6(\mathrm{C}-8)]$, an oxygenated methine $\left[\delta_{\mathrm{H}} 3.62(1 \mathrm{H}, \mathrm{m}, \mathrm{H}-9) ; \delta_{\mathrm{C}} 66.3\right]$ and a methyl group $\left[\delta_{\mathrm{H}} 1.11(3 \mathrm{H}, \mathrm{d}, J=6.0 \mathrm{~Hz}, \mathrm{H}-10) ; \delta_{\mathrm{C}} 22.1\right]$ were observed in ${ }^{1} \mathrm{H}$ - and ${ }^{13} \mathrm{C}-\mathrm{NMR}$ together with the HSQC spectrum. The HMBC correlations of H-2/C7, H-8/C-9 and H-10/C-9 attributed the connection of a 1-(3,5-dihydroxyphenyl)-butan-3-ol group to the glucose and trans-caffeoyl groups. The HMBC correlations of $\mathrm{H}-1^{\prime} / \mathrm{C}-3$ and of $\mathrm{H}-6^{\prime} / \mathrm{C}-9^{\prime \prime}$ confirmed the linkage between a trans-caffeoyl group, a glucose and a 1-(3,5-dihydroxyphenyl)-butan-3-ol group, as shown in Figure 2. Conclusively, compound 4 was defined as shown and named argutoside D.

Compound 5 (Table 3) was purified as a brown syrup with the molecular formula of $\mathrm{C}_{24} \mathrm{H}_{22} \mathrm{O}_{12}$ deduced by HRESI-TOF-MS analysis $\left(\mathrm{m} / \mathrm{z} 525.1003\right.$, calcd. for $\mathrm{C}_{24} \mathrm{H}_{22} \mathrm{NaO}_{12}$, 525.1009) and ${ }^{13} \mathrm{C}-\mathrm{NMR}$ data. The ${ }^{1} \mathrm{H}$ - and ${ }^{13} \mathrm{C}-\mathrm{NMR}$ spectra of compound 5 showed the signals for a glucose and a trans-coumaroyl group, similar to those of compounds 1-4. However, signals for two cis-olefinic protons at $\delta_{\mathrm{H}} 7.68(1 \mathrm{H}, \mathrm{d}, J=9.2 \mathrm{~Hz}, \mathrm{H}-4)$ and 5.85 $(1 \mathrm{H}, \mathrm{d}, J=9.2 \mathrm{~Hz}, \mathrm{H}-3)$ suggested that compound 5 was a coumarin derivative, which was supported by the characteristic UV absorption maxima at 211 and $327 \mathrm{~nm}$. Additionally, two aromatic protons at $\delta_{\mathrm{H}} 7.24(1 \mathrm{H}, \mathrm{s}, \mathrm{H}-5)$ and $\delta_{\mathrm{H}} 6.74(1 \mathrm{H}, \mathrm{s}, \mathrm{H}-8)$ together with ${ }^{13} \mathrm{C}-\mathrm{NMR}$ signals of $\delta_{\mathrm{C}} 166.8$ (C-2), 111.7 (C-3), 144.7 (C-4), 110.6 (C-4a), 114.7 (C-5), 151.1 (C-6), 143.2 (C-7), 103.7 (C-8), 146.1 (C-8a)] suggested the existence of one 6,7-disubstituted coumarin skeleton. Further analysis using the HMBC correlation together with a comparison to the previous data identified the coumarin moiety as 6,7-dihydroxycoumarin, esculetin [22]. Therefore, compound 1 was suggested to consist of a glucose, a trans-caffeoyl group and a 6,7-dihydroxycoumarin moiety. The HMBC correlations from $\mathrm{H}-1^{\prime}$ of a glucose to C-7 of a 6,7-dihydroxycoumarin group suggested the linkage between 6,7-dihydroxycoumarin and a glucose, and the HMBC correlations from H-6' of a glucose to C-9" of trans-caffeoyl group suggested the linkage between a glucose and a trans-caffeoyl group. Based on these data, the structure of compound 5 was defined as esculetin 7-O-(6'-O-trans-caffeoyl)- $\beta$ glucopyranoside and named argutoside $\mathrm{E}$. 
Table $3 .{ }^{1} \mathrm{H}$ - and ${ }^{13} \mathrm{C}-\mathrm{NMR}$ spectroscopic data for compound 5 (DMSO- $d_{6}$ ).

\begin{tabular}{ccc}
\hline Carbon NO. & ${ }^{\mathbf{1}} \mathbf{H}$ & ${ }^{3} \mathbf{C}$ \\
\hline 2 & - & 166.8 \\
3 & $7.85(\mathrm{~d}, 9.2)$ & 111.7 \\
4 & $-68(\mathrm{~d}, 9.2)$ & 144.7 \\
$4 \mathrm{a}$ & $7.24(\mathrm{~s})$ & 110.6 \\
5 & - & 114.7 \\
6 & - & 151.1 \\
7 & $6.74(\mathrm{~s})$ & 143.2 \\
8 & - & 103.7 \\
$8 \mathrm{a}$ & $4.81(\mathrm{~d}, 7.2)$ & 146.1 \\
$1^{\prime}$ & $3.34-3.50(\mathrm{~m})$ & 102.3 \\
$2^{\prime}$ & $3.34-3.50(\mathrm{~m})$ & 73.6 \\
$3^{\prime}$ & $3.34-3.50(\mathrm{~m})$ & 76.3 \\
$4^{\prime}$ & $3.68(\mathrm{~m})$ & 70.5 \\
$5^{\prime}$ & - & 74.5 \\
$6^{\prime}$ & $7.06(\mathrm{~d}, 1.6)$ & 63.8 \\
$1^{\prime \prime}$ & - & 125.9 \\
$2^{\prime \prime}$ & - & 115.4 \\
$3^{\prime \prime}$ & $6.77(\mathrm{~d}, 8.0)$ & 146.1 \\
$4^{\prime \prime}$ & $6.99(\mathrm{dd}, 8.0,1.6)$ & 149.0 \\
$5^{\prime \prime}$ & $7.49(\mathrm{~d}, 16.0)$ & 116.2 \\
$6^{\prime \prime}$ & $6.32(\mathrm{~d}, 16.0)$ & 121.9 \\
$7^{\prime \prime}$ & - & 145.8 \\
$8^{\prime \prime}$ & & 114.3 \\
$9^{\prime \prime}$ & $4.45(\mathrm{dd}, 12.0,1.6)$ & 166.8 \\
\hline
\end{tabular}

\subsubsection{Identification of Known Compounds}

The known compounds were identified as esculetin 7-O-(6'-O-trans-coumaroyl)- $\beta$ glucopyranoside (6) [23], umbelliferone 7-O-(6'-O-trans-coumaroyl)- $\beta$-glucopyranoside (7) [23], esculetin (8) [22], 7,8-Dihydroxycoumarin (9) [24], umbelliferone (10) [25], 4hydroxybenzoic acid (11) [26], protocatechuic acid (12) [26], vanillic acid (13) [27], isovanillic acid (14) [28], hydroxytyrosol (15) [28], (-)-rhodolatouchol (16) [29], $p$-E-coumaric acid (17) [30], $p$-E-coumaric acid-9-O-glucopyranoside (18) [23], E-caffeic acid (19) [28], E-ferulic acid (20) [31], 3,5-dimethoxy-4-hydroxycinnamic alcohol (21) [32], kaempferol (22) [33], kaempferol 3-O- $\beta$-glucopyranoside (23) [34], kaempferol 3-O- $\beta$-galactopyranoside (24) [35], quercetin (25) [36], tamarixin (26) [37], isorhamnetin 3-O- $\beta$-glucopyranoside (27) [30], rhamnetin 3-O- $\beta$-glucopyranoside (28) [35], dihydrokaempferol (29) [35], dihydroquercetin (30) [38], naringenin (31) [35], 5,7,3',5'-tetrahydroxyflavanone (32) [38], sinensin (33) [39], quercetin 3-O-(6"-O-E-caffeoyl)- $\beta$-glucopyranoside (34) [39], quercetin 3$O-(6 "$ "-O-E-coumaroyl)- $\beta$-glucopyranoside (35) [40], epicatechin (36) [30], catechin (37) [35], cinchonain Ia (38) [41], catechin-[8,7-e]-4b-(3,4-dihydroxy-phenyl)-dihydro-2(3H)-pyranone (39) [40], 7S,8R-cedrusin (40) [42], dehydroconiferyl alcohol (41) [40], (7S,8S)-3-methoxy$3^{\prime}, 7$-epoxy-8, $4^{\prime}$-oxyneoligna-4,9,9'-triol (42) [43], pinoresinol (43) [44], pinoresinol 4-O- $\beta$ glucopyranoside (44) [42], alutaceuol (45) [29], alutaceuol isomer (46) [29], (-)-(2R,3R)secoisolariciresinol (47) [45], glehlinoside F (48) [46] via analysis of their physical data and comparison with literature values.

\subsection{Antioxidant and $\alpha$-Glucosidase Inhibitory Activity}

\subsubsection{Antioxidant and $\alpha$-Glucosidase Inhibitory Activity of Compounds}

The antioxidant and anti-diabetic activity of the isolated compounds were evaluated by measuring the DPPH radical scavenging and $\alpha$-glucosidase inhibitory activity. The isolated compounds showed good antioxidant and $\alpha$-glucosidase inhibitory activity with differences in activity depending on the structures. In particular, new compounds 1, 2, 4 and 5 showed antioxidant activity and compounds 1, 3 and 5 showed $\alpha$-glucosidase inhibitory activity in our assay system (Figure 3). 


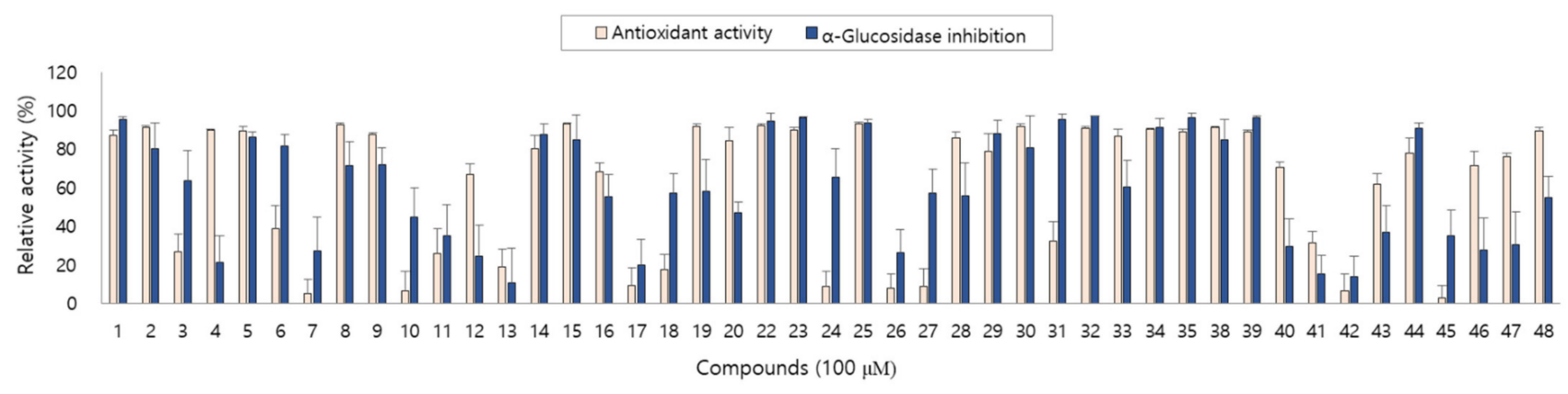

Figure 3. Antioxidant and $\alpha$-glucosidase inhibitory activity of compounds 1-48 from A. arguta leaves.

As described above, the leaf of A. arguta is rich in phenolic compounds, and a total of 48 compounds were purified in this study. All 48 of the compounds that were isolated in this study are aromatic compounds and can be subdivided according to the compound skeleton as follows: phenylpropanoid derivatives $(\mathbf{1}-\mathbf{4}, \mathbf{1 6}-\mathbf{2 1})$, coumarins (5-10), simple phenolics (11-15), flavonoids (22-39) and lignans (40-48). The biological activity of the isolated compounds differs depending on their structure, and flavonoid showed excellent efficacy while lignan showed comparatively weak efficacy in our assay system. Interestingly, the leaf of $A$. arguta contained phenolic-conjugates that were bound to various skeletons such as phenylpropanoid-conjugates, coumarin-conjugates, flavonoid-conjugates and lignanconjugates. These conjugates showed antioxidant and $\alpha$-glucosidase inhibitory activity and contributed to the beneficial effect of $A$. arguta leaves.

\subsubsection{Molecular Docking Analysis}

Further molecular docking analysis was conducted for two types of human maltaseglucoamylase (NtMGAM and CtMGAM) in order to propose the mechanisms of the $\alpha$ glucosidase inhibitory activity of active compounds. Consistent with experimental results, interactions with the $\alpha$-glucosidase were suggested for active compounds. Hydrogen bonds were formed between compound 1 and NtMGAM and CtMGAM, respectively. Compound 5 exhibited the interaction by forming hydrogen bonds and Pi-alkyl interactions, as shown in Figure 4. These results indicate that compounds $\mathbf{1}$ and $\mathbf{5}$ could be inserted into the active site of the enzyme by different types of interactions and could inhibit $\alpha$-glucosidase activity.
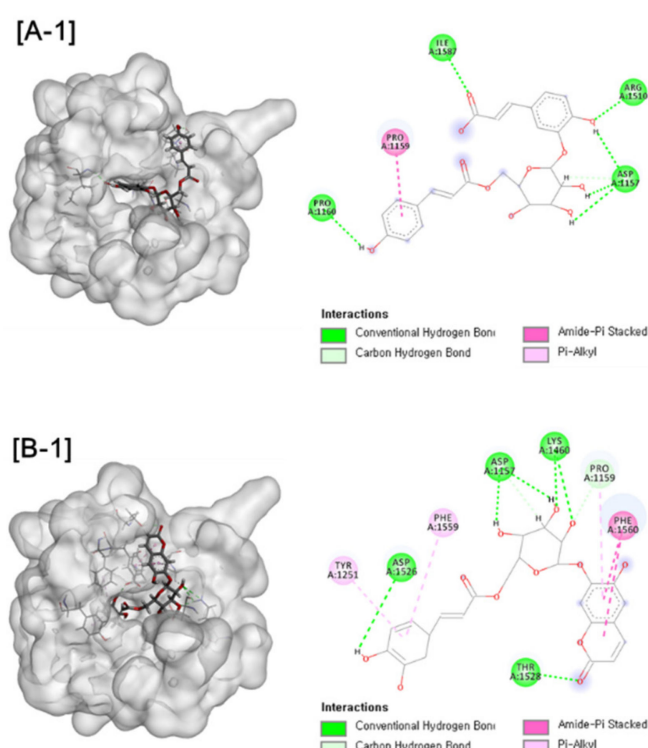

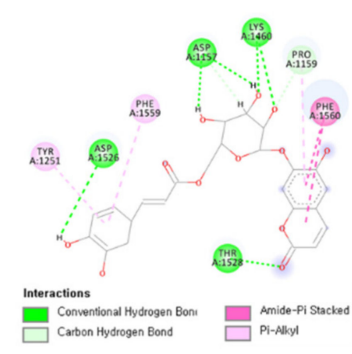

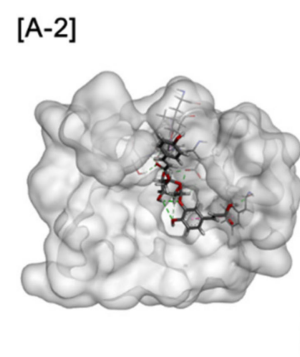

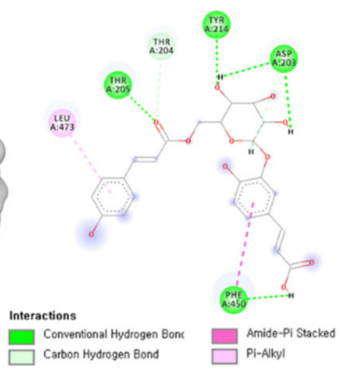

[B-2]

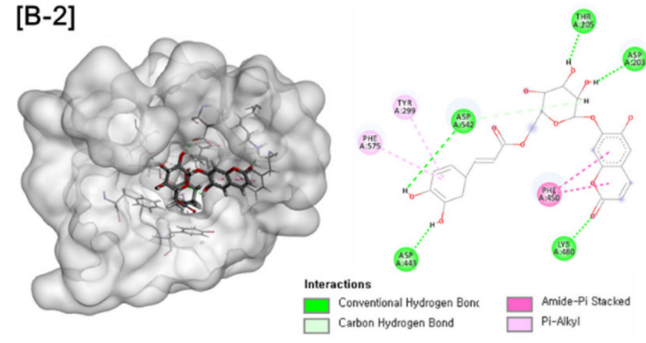

Figure 4. [A] Docking picture of compound 1 to CtMGAM (A-1) and NtMGAM (A-2) and [B] compound 5 to CtMGAM (B-1) and NtMGAM (B-2). The interactions of conventional hydrogen bond (green color), carbon hydrogen bond (light green color), amide-Pi stacked (pink color) and Pi-alkyl (light pine) were shown. 


\section{Conclusions}

An investigation into the leaves of $A$. arguta led to the isolation of 48 aromatic compounds, including 5 new compounds. The structures of the isolated compounds were determined to be aromatic, including phenylpropanoid derivatives, phenolics, coumarins, flavonoids and lignans. Five new compounds were defined as argutosides A-D (1-4), which consist of phenylpropanoid glycosides conjugated with a phenolic compound, and argutosides E (5), which is a coumarin glycoside conjugated with a phenylpropanoid. The isolated compounds showed good antioxidant and $\alpha$-glucosidase inhibitory activity with differences in activity depending on the structures. The analysis of the interactions between hydroxyl and carbonyl groups of active compounds $\mathbf{1}$ and $\mathbf{5}$ and $\alpha$-glucosidase by molecular docking analysis supported the $\alpha$-glucosidase inhibitory activity. In conclusion, the aromatic constituents of $A$. arguta leaves with $\alpha$-glucosidase inhibitory activity might be beneficial to glucose-related diseases.

Supplementary Materials: The following are available online at https:/ / www.mdpi.com/article/10 .3390 / antiox10121896/s1, Figures S1-S25: ${ }^{1} \mathrm{H},{ }^{13} \mathrm{C}, \mathrm{HSQC}, \mathrm{HMBC}$ and HRESI-MS spectrums of new compounds 1-5.

Author Contributions: Conceptualization, J.H.A. and M.K.L.; methodology, J.H.A., S.H.R., S.L., S.W.Y. and A.T.; software, Y.K.H. and K.Y.L.; validation, J.H.A., S.H.R., B.Y.H. and M.K.L.; formal analysis, J.H.A., B.Y.H. and M.K.L.; investigation, J.H.A., S.H.R., S.L., S.W.Y., A.T. and M.K.L.; writing - original draft preparation, J.H.A., S.H.R. and M.K.L.; writing—-review and editing, J.H.A., S.H.R. and M.K.L.; supervision, M.K.L.; project administration, M.K.L.; funding acquisition, M.K.L. All authors have read and agreed to the published version of the manuscript.

Funding: This work was supported by Basic Science Research Program (2018R1D1A1A09082613) and Medical Research Center program (2017R1A5A2015541) through the National Research Foundation of Korea.

Institutional Review Board Statement: Not applicable.

Informed Consent Statement: Not applicable.

Data Availability Statement: The data is contained within the article or Supplementary Material.

Acknowledgments: The authors wish to thank the Korea Basic Science Institute for the NMR spectroscopic measurements.

Conflicts of Interest: The authors declare no conflict of interest.

\section{References}

1. Reuter, S.; Gupta, S.C.; Chaturvedi, M.M.; Aggarwal, B.B. Oxidative stress, inflammation and cancer: How are they linked? Free Radic. Biol. Med. 2010, 49, 1603-1616. [CrossRef] [PubMed]

2. Butterfield, D.A.; Halliwell, B. Oxidative stress, dysfunctional glucose metabolism and Alzheimer disease. Nat. Rev. Neurosci. 2019, 20, 148-160. [CrossRef] [PubMed]

3. Yaribeygi, H.; Sathyapalan, T.; Atkin, S.L.; Sahebkar, A. Molecular mechanisms linking oxidative stress and diabetes mellitus. Oxid. Med. Cell Longev. 2020, 2020, 8609213. [CrossRef] [PubMed]

4. Brownlee, M. The pathobiology of diabetic complications a unifying mechanism. Diabetes 2005, 54, 1615-1625. [CrossRef] [PubMed]

5. Pitocco, D.; Tesauro, M.; Alessandro, R.; Ghirlanda, G.; Cardillo, C. Oxidative stress in diabetes: Implications for vascular and other complications. Int. J. Mol. Sci. 2013, 14, 21525-21550. [CrossRef]

6. Giacco, F. Oxidative stress and diabetic complications. Circ. Res. 2011, 107, 1058-1070. [CrossRef]

7. Yao, D.; Brownlee, M. Hyperglycemia-induced reactive oxygen species increase expression of the receptor for advanced glycation end products (RAGE) and RAGE ligands. Diabetes 2010, 59, 249-255. [CrossRef]

8. Stefano, G.B.; Challenger, S.; Kream, R.M. Hyperglycemia-associated alterations in cellular signaling and dysregulated mitochondrial bioenergetics in human metabolic disorders. Eur. J. Nutr. 2016, 55, 2339-2345. [CrossRef]

9. Maritim, A.C.; Sanders, R.A.; Watkins, J.B. Diabetes, oxidative stress, and antioxidants: A review. J. Biochem. Mol. Toxicol. 2003, 17, 24-38. [CrossRef]

10. Rendra, E.; Riabov, V.; Mossel, D.M.; Sevastyanova, T.; Harmsen, M.C.; Kzhyshkowska, J. Reactive oxygen species (ROS) in macrophage activation and function in diabetes. Immunobiology 2018, 224, 242-253. [CrossRef] 
11. Ghani, U. Re-exploring promising $\alpha$-glucosidase inhibitors for potential development into oral anti-diabetic drugs: Finding needle in the haystack. Eur. J. Med. Chem. 2015, 103, 133-162. [CrossRef]

12. Joshi, S.R.; Standl, E.; Tong, N.; Shah, P.; Kalra, S.; Rathod, R. Therapeutic potential of $\alpha$-glucosidase inhibitors in type 2 diabetes mellitus: An evidence-based review. Expert Opin. Pharmacother. 2015, 16, 1959-1981. [CrossRef]

13. Umeno, A.; Horie, M.; Murotomi, K.; Nakajima, Y.; Yoshida, Y. Antioxidative and antidiabetic effects of natural polyphenols and isoflavones. Molecules 2016, 30, 708. [CrossRef]

14. Lin, D.; Xiao, M.; Zhao, J.; Li, Z.; Xing, B.; Li, X.; Kong, M.; Li, L.; Zhang, Q.; Liu, Y.; et al. An Overview of plant phenolic compounds and their importance in human nutrition and management of type 2 diabetes. Molecules 2016, 21, 1374. [CrossRef]

15. Zhang, P.; Li, T.; Wu, X.; Nice, E.C.; Huang, C.; Zhang, Y. Oxidative stress and diabetes: Antioxidative strategies. Front. Med. 2020, 14, 583-600. [CrossRef]

16. Latocha, P. The nutritional and health benefits of kiwiberry (Actinidia arguta)—A Review. Plant Foods Hum. Nutr. 2017, 72, 325-334. [CrossRef]

17. Almeida, D.; Pinto, D.; Santos, J.; Vinha, A.F.; Palmeira, J.; Ferreira, H.N.; Rodrigues, F.; Oliveira, B.P.P. Hardy kiwifruit leaves (Actinidia arguta): An extraordinary source of value-added compounds for food industry. Food Chem. 2018, 259, 113-121. [CrossRef]

18. Kim, H.Y.; Hwang, K.W.; Park, S.Y. Extracts of Actinidia arguta stems inhibited LPS-induced inflammatory responses through nuclear factor- $\mathrm{kB}$ pathway in Raw 264.7 cells. Nutr. Res. 2014, 34, 1008-1016. [CrossRef]

19. Heo, K.H.; Sun, X.; Shim, D.W.; Kim, M.K.; Koppula, S.; Yu, S.H.; Kim, H.B.; Kim, T.J.; Kang, T.B.; Lee, K.H. Actinidia arguta extract attenuates inflammasome activation: Potential involvement in NLRP3 ubiquitination. J. Ethnopharmacol. 2018, 213, 159-165. [CrossRef]

20. Kim, G.D.; Lee, J.Y.; Auh, J.H. Metabolomic screening of anti-inflammatory compounds from the leaves of Actinidia arguta (Hardy Kiwi). Foods 2019, 8, 47. [CrossRef]

21. Ahn, J.H.; Park, Y.; Yeon, S.W.; Jo, Y.H.; Han, Y.K.; Turk, T.; Ryu, S.H.; Hwang, B.Y.; Lee, K.Y.; Lee, M.K. Phenylpropanoidconjugated triterpenoids from the leaves of Actinidia arguta and their inhibitory activity on $\alpha$-glucosidase. J. Nat. Prod. 2020, 83, 1416-1423. [CrossRef] [PubMed]

22. He, R.; Huang, X.; Zhang, Y.; Wu, L.; Nie, H.; Zhou, D.; Liu, B.; Deng, S.; Yang, R.; Huang, S.; et al. Structural characterization and assessment of the cytotoxicity of 2,3-dihydro-1H-indene derivatives and coumarin glucosides from the bark of Streblus indicus. J. Nat. Prod. 2016, 79, 2472-2478. [CrossRef] [PubMed]

23. Ahn, J.H.; Park, Y.; Jo, Y.H.; Kim, S.B.; Yeon, S.W.; Kim, J.G.; Turk, A.; Song, J.Y.; Kim, Y.; Hwang, B.Y.; et al. Organic acid conjugated phenolic compounds of hardy kiwifruit (Actinidia arguta) and their NF-kB inhibitory activity. Food Chem. 2020, 308, 125666. [CrossRef] [PubMed]

24. Lin, Y.L.; Wang, W.Y. Chemical constituents of Vernonia patula. Chin. Pharm. J. 2002, 54, 187-192.

25. Ma, B.; Guo, H.F.; Lou, H.X. A new lignan and two eudesmanes from Lepidozia vitrea. Helvet. Chim. Acta 2007, 90, 58-62. [CrossRef]

26. Timonen, J.M.; Nieminen, R.M.; Sareila, O.; Goulas, A.; Moilanen, L.J.; Haukka, M.; Vainiotalo, P.; Moilanen, E.; Aulaskari, P.H. Synthesis and anti-inflammatory effects of a series of novel 7-hydroxycoumarin derivatives. Eur. J. Med. Chem. 2011, 46, 3845-3850. [CrossRef]

27. Ding, H.Y.; Lin, H.C.; Teng, C.M.; Wu, Y.C. Phytochemical and pharmacological studies on Chinese Paeonia species. J. Chin. Chem. Soc. 2000, 47, 381-388. [CrossRef]

28. Hoang, L.; Joo, G.J.; Kim, W.C.; Jeon, S.Y.; Choi, S.H.; Kim, J.W.; Rhee, I.K.; Hur, J.M.; Song, K.S. Growth inhibitors of lettuce seedings from Bacillus cereus EJ-121. Plant Growth Reg. 2005, 47, 149-154. [CrossRef]

29. Park, C.H.; Kim, K.H.; Lee, I.K.; Lee, S.Y.; Choi, S.U.; Lee, J.H.; Lee, K.R. Phenolic constituents of Acorus gramineus. Arch. Pharm. Res. 2011, 34, 1289-1296. [CrossRef]

30. Li, H.Z.; Song, H.J.; Li, H.M.; Pan, Y.Y.; Li, R.T. Characterization of phenolic compounds from Rhododendron alutaceum. Arch. Pharm. Res. 2012, 35, 1887-1893. [CrossRef]

31. Lee, Y.G.; Cho, J.Y.; Kim, C.M.; Lee, S.H.; Kim, W.S.; Jeon, T.I.; Park, K.H.; Moon, J.H. Coumaroyl quinic acid derivatives and flavonoids from immature pear (Pyrus pyrifolia Nakai) fruit. Food Sci. Biotechnol. 2013, 22, 803-810. [CrossRef]

32. Prachayasittikul, S.; Suphapong, S.; Worachartcheewan, A.; Lawung, R.; Ruchirawat, S.; Prachayasittikul, V. Bioacitive metabolites from Spilanthes acmella Murr. Molecules 2009, 14, 850-867. [CrossRef]

33. Bohlmann, F.; Chen, Z.L.; Schuster, A. Aromatic esters from Solidago decurrens. Phytochemstry 1981, 20, 2601-2602. [CrossRef]

34. Itoh, T.; Ninomiya, M.; Yasuda, M.; Koshikawa, K.; Deyashiki, Y.; Nozawa, Y.; Akao, Y.; Koketsu, M. Inhibitory effects of flavonoids isolated from Fragaria ananassa Duch on IgE-mediated degranulation in rat basophilic leukemia RBL-2H3. Bioorg. Med. Chem. 2009, 17, 5374-5379. [CrossRef]

35. Han, J.T.; Bang, M.H.; Chun, O.K.; Kim, D.O.; Lee, C.Y.; Baek, N.I. Flavonol glycosides from the aerial parts of Aceriphyllum rossii and their antioxidant activities. Arch. Pharm. Res. 2004, 27, 390-395. [CrossRef]

36. Jeon, S.H.; Chun, W.J.; Choi, Y.J.; Kwon, Y.S. Cytotoxic constituents from the bark of Salix hulteni. Arch. Pharm. Res. 2008, 31, 978-982. [CrossRef]

37. Lim, E.K.; Ashford, D.A.; Hou, B.K.; Jackson, R.G.; Bowles, D.J. Arabidopsis glycosyltransferases as biocatalysts in fermentation for regioselective synthesis of diverse quercetin glucosides. Biotechnol. Bioeng. 2004, 87, 623-631. [CrossRef] 
38. Fico, G.; Rodondi, G.; Flamini, G.; Passarella, D.; Tome, F. Comparative phytochemical and morphological analyses of three Italian Primula species. Phytochemistry 2007, 68, 1683-1691. [CrossRef]

39. He, Z.; Lian, W.; Liu, J.; Zheng, R.; Xu, H.; Du, G.; Liu, A. Isolation, structural characterization and neuraminidase inhibitory activities of polyphenolic constituents from Flos caryophylli. Phytochem. Lett. 2017, 19, 160-167. [CrossRef]

40. Calzada, F.; Cedillo-Rivera, R.; Mata, R. Antiprotozoal activity of the constituents of Conyza filaginoides. J. Nat. Prod. 2001, 64, 671-673. [CrossRef]

41. Wan, C.P.; Yuan, T.; Cirello, A.L.; Seeram, N.P. Antioxidant and $\alpha$-glucosidase inhibitory phenolics isolated from highbush blueberry flowers. Food Chem. 2012, 135, 1929-1937. [CrossRef] [PubMed]

42. Pizzolatti, M.G.; Venson, A.F.; Junior, A.S.; Smania, E.F.A.; Braz-Filho, R. Two epimeric flavalignans from Trichilia catigua (Meliaceae) with antimicrobial activity. J. Biosci. 2002, 57, 483-488. [CrossRef] [PubMed]

43. Kim, T.H.; Ito, H.; Hayashi, K.; Hasegawa, T.; Machiguchi, T.; Yoshida, T. Aromatic constituents from the Heartwood of Santalum album L. Chem. Pharm. Bull. 2005, 53, 641-644. [CrossRef] [PubMed]

44. Fang, J.M.; Lee, C.K.; Cheng, Y.S. Lignans from leaves of Juniperus chinensis. Phytochemistry 1992, 31, 3659-3661.

45. Kang, W.; Wang, J. In vitro antioxidant properties and in vivo lowering blood lipid of Forsythia suspense leaves. Med. Chem. Res. 2010, 19, 617-628. [CrossRef]

46. Moon, S.S.; Rahman, A.A.; Kim, J.Y.; Kee, S.H. Hanultarin, a cytotoxic lignin as an inhibitor of actin cytoskeleton polymerization from the seeds of Trichosanthes kirilowii. Bioorg. Med. Chem. 2008, 16, 7264-7269. [CrossRef] 